\title{
Electronic medication management: is it a silver bullet?
}

\author{
Robert Pearce \\ Project manager \\ Electronic Medications \\ Management \\ Information Technology and \\ Telecommunications \\ Hunter New England Local \\ Health District

\section{Ian Whyte} \\ Director \\ Clinical Toxicology and \\ Pharmacology \\ Calvary Mater Newcastle \\ Hunter New England Local \\ Health District \\ New South Wales
}

\section{Keywords}

electronic prescribing,

medication errors

Aust Prescr 2018;41:32-3

https://doi.org/10.18773/ austprescr.2018.012
Electronic medication management has been developed to improve patient safety by increasing the legibility of prescriptions, implementing passive and active decision support and allowing access to medical records across a wide area. It is a patient safety initiative, albeit some stakeholders see it as a cost-saving exercise. Electronic medication management is a broad term covering all computer systems involved. It is a closed loop system that encompasses prescribing, administration, pharmacy review, smart infusion pumps, automated dispensing cabinets, barcode medication administration and anything that has electronic medicines datasets or encompasses medication management processes. ${ }^{2}$

There are a number of electronic medication systems available. These vary from software for individual practitioners to stand-alone systems for specialties (e.g. oncology, intensive care), and hospital or districtwide systems with or without an integrated, fully electronic medical record.

Electronic prescribing keeps track of medicine use through computer applications. With districtwide systems, this record is available across all sites within the local health district for transfers or future admissions. For example, when a patient is discharged from a hospital in NSW, the discharge script is printed from the software and the discharge medication list is exported in the electronic discharge summary for transmission to GPs, NSW HealtheNet and My Health Record.

An electronic prescribing system provides an easily accessible record of administration. It improves access to medication histories across the continuum of care from the GP to the hospital and back to the GP.

Data and reports available in electronic prescribing systems allow audits on drug use, including tracking orders for antimicrobial stewardship, medicine recalls and analysis of usage patterns. The software can incorporate standardised prescribing protocols for specific conditions, for example pain management, vaccinations and acute coronary syndromes.

Responses to drug shortages across a health district can be coordinated with alerts and suggestions for alternatives. These can be deployed across all sites rapidly.

One of the main benefits of using an electronic prescribing system is that the software improves overall legibility. A clear, typed prescription decreases interpretive errors. The person reviewing or administering does not need to decipher illegible handwriting and error-prone abbreviations.

Electronic prescribing also reduces the risk of dosing errors as it can specify commonly used doses. Potentially dangerous doses are minimised as the software guides prescribers towards using the more common doses. This does not, however, prevent all of these errors as the software needs to provide flexibility during prescribing. For example, restricting oral methotrexate to a maximum of $30 \mathrm{mg}$ on one day per week would prevent prescribing of the higher doses (up to $100 \mathrm{mg}$ ) required for medical management of ectopic pregnancy.

Recording of electronic prescribing and administration also allows the standardisation of orders and can provide clinical alerts. For example, if clozapine is prescribed, a message to contact the clozapine coordinator can appear along with the investigations required for safe prescribing.

Electronic prescribing can also help to prevent the prescribing of drugs to patients with medication allergies by recording allergy and adverse drug reaction information. When allergies are correctly entered into the system, the software alerts the prescriber with details of the allergy. Unfortunately, even with prompts within the system, not all patients have their allergies recorded, some allergies are recorded incorrectly, and in larger organisations there may be multiple silos of allergy data with details recorded in other sections of the patient record that are not accessible to the electronic prescribing system.

Another issue with managing allergies is that it does not prevent clinicians from entering incorrect information (simple user error). For example, a prescriber could enter penicillamine allergy for a substance allergy when they mean penicillin. In the electronic prescribing software, the specific substance is benzylpenicillin or phenoxymethylpenicillin and the allergy group is penicillin.

With any electronic prescribing system comes an opportunity to provide knowledge-based clinical decision support at the time of prescribing or administration. However, this needs to be balanced with the risk of alert fatigue. ${ }^{3}$ The commonest source of alerts is for drug interactions. Unfortunately, in 
some systems the drug interactions detected can be over-inclusive, and trivial or minor potential interactions can fire the same or very similar styles of alert as potentially life-threatening ones. As a result, the flurry of much more common, unimportant alerts trains the user to ignore all alerts including the important ones.

Another frequent source of alerts is therapeutic duplication which warns if two drugs of the same class are prescribed simultaneously. Here, the usefulness of such alerts depends heavily on the definitions of the therapeutic classes.

If, for example, all corticosteroids are put into one class then an alert will fire (inappropriately) when a patient with asthma on preventative puffers is prescribed prednisolone for an acute exacerbation. Having heparins and oral anticoagulants in one therapeutic class will provide an appropriate alert when enoxaparin is inadvertently prescribed as bridging therapy for a patient starting on rivaroxaban, but an inappropriate alert if that patient was starting on warfarin. A warning regarding multiple antipsychotics may be important to a junior medical officer on a general medical ward, but to a psychiatrist in a mental health unit it may be annoying.

In our own implementation of an electronic prescribing system for the Hunter New England Local Health District, the Quality Use of Medicines
Committee turned off drug interaction warnings on the advice of sites already using the software. However, we have continued the alerts for therapeutic duplication. Our preliminary analysis shows that 95\% of duplicate therapy warnings are not immediately actioned. However, what about the 5\% that are actioned? Has this made a significant impact? ${ }^{4}$ In a system as complex as a large heath district, it is difficult to isolate cause and effect. The Quality Use of Medicines Committee has decided to remove duplicate therapy warnings provided by the software vendor and replace them with specific tailored alerts determined by local expertise. The goal is to make every alert relevant to that prescribing or administration circumstance. As the software matures, we hope to nuance our warnings and alerts.

The implementation of electronic prescribing across our district has improved patient safety, communication and accountability, and provides an electronic record of medication prescribing and administration. However, ongoing work needs to be done to address problems with alerts, developing protocols, adding new medications and overall system improvements. We need to improve usability to increase engagement while maintaining the focus on patient safety. $<$

Conflict of interest: none declared

\section{REFERENCES}

1. Westbrook JI, Reckmann M, Li L, Runciman WB, Burke R, Lo $C$, et al. Effects of two commercial electronic prescribing systems on prescribing error rates in hospital in-patients: a before and after study. PLoS Med 2012;9:e1001164. https://doi.org/10.1371/journal.pmed.1001164

2. Franklin BD, O'Grady K, Donyai P, Jacklin A, Barber N. The impact of a closed-loop electronic prescribing and administration system on prescribing errors, administration errors and staff time: a before-and-after study. Qual Saf Health Care 2007;16:279-84. https://doi.org/10.1136/ qshc.2006.019497
3. Footracer KG. Alert fatigue in electronic health records. JAAPA 2015;28:41-2. https://doi.org/10.1097/ 01.JAA.0000465221.04234.ca

4. Genco EK, Forster JE, Flaten H, Goss F, Heard KJ, Hoppe J, et al. Clinically inconsequential alerts: the characteristics of opioid drug alerts and their utility in preventing adverse drug events in the emergency department. Ann Emerg Med 2016;67:240-248.e3. https://doi.org/10.1016/ j.annemergmed.2015.09.020 